\title{
Isotropic-nematic transition of hard ellipses
}

\author{
J. A. Cuesta and C. F. Tejero \\ Física Aplicada I, Facultad de Ciencias Físicas, Universidad Complutense de Madrid, 28040 Madrid, Spain \\ M. Baus \\ Faculté des Sciences, Case Postale 231, Université Libre de Bruxelles, B-1050 Brussels, Belgium
}

(Received 27 January 1989)

\begin{abstract}
The orientational freezing of a system of hard ellipses, as a first approximation for a nematogen adsorbed on a smooth substrate, is studied with the aid of an approximate density-functional theory used previously for the study of hard ellipsoids. The isotropic-nematic transition, which was first order for the ellipsoids, is shown to proceed via a continuous transition in the case of the ellipses. We also show that when reducing the dimensionality of the angular space of ellipsoids, the width of the transition shrinks continuously and reaches zero only for a strictly two-dimensional angular space.
\end{abstract}

\section{INTRODUCTION}

The formation of liquid crystals or mesophases by molecular systems is well known ${ }^{1}$ to be closely related to the presence of anisotropic interactions between the molecules. One of the most important findings of the computer simulations ${ }^{2}$ and theoretical studies ${ }^{3}$ of recent years is the increasing evidence that it is sufficient to have anisotropic repulsive (steric) interactions in order to form such mesophases. This contradicts some of the early ideas ${ }^{4}$ which stressed the importance of the anisotropic attractive interactions in liquid crystals forming substances. The present situation is thus very reminiscent of the older debate $^{5}$ concerning the respective roles played by the repulsive and attractive forces in the liquid-solid transition. Nowadays it is, nevertheless, generally accepted ${ }^{5}$ that this freezing transition is monitored by the repulsive forces and a similar issue is in view for the liquid-crystal phase transitions. This, however, does not imply that the attractive forces do not play any role in these phase transitions but only that the essence of these transitions can already be learned from the study of simple nonrealistic models with purely repulsive forces. Such model systems can then be used as reference systems ${ }^{5}$ in a perturbational treatment of the more realistic systems.

From all possible purely repulsive model systems, those where the molecules are assimilated to hard convex bodies are by far the simplest and most-studied ones. It is, for example, known for more than two decades that the hard-sphere system is a convenient reference system for the study of the condensed phases of spherically symmetric molecules. Similarly, a system of hard ellipsoids or spherocylinders appears to be a convenient reference system ${ }^{2,3}$ for the study of nonspherical convex molecules (more complex molecular shapes can also be introduced by using the simpler shapes as building blocks). Much progress in our understanding of the behavior of realistic systems can thus be expected from the study of such hard-core model systems. This is certainly true for the bulk phases of matter but a similar reduction process ap- pears to be feasible also for the surface-phase transitions experienced by monolayers of adsorbed molecules. A system of hard disks, for example, is known ${ }^{5}$ to capture the essence of the liquid-solid transition of spherical molecules adsorbed on a smooth surface. In the present study we consider similarly a system of hard ellipses as a simple model for the study of the mesophases ${ }^{6}$ formed by nonspherical molecules adsorbed on a smooth surface. ${ }^{7}$

It is well known ${ }^{8}$ that the reduction in spatial dimensionality from $D=3$ (bulk phases) to $D=2$ (surface phases) has some profound consequences for the nature of the ordered phases. Reducing the dimensionality usually increases the importance of the thermally excited long-wavelength elastic waves which tend to destroy the perfect long-range order leaving one with quasi-phases whose order exhibits a system-size dependence. ${ }^{8}$ This size dependence is usually sufficiently weak (logarithmic in $D=2$ ) that for many practical purposes the difference between quasi long-range order and perfect long-range order can be neglected for most systems of laboratory interest. The algebraic decay of the order-parameter correlation function associated with the presence of quasilong-range order has nevertheless been observed recently in computer simulations ${ }^{9}$ and laboratory experiments. ${ }^{10}$ Such system-size effects are, however, also beyond the scope of the present-day theories of phase transitions ${ }^{11}$ and will therefore be neglected in what follows.

We now consider the isotropic and nematic phases of a two-dimensional system of hard ellipses as a rough approximation for the isotropic-nematic transition of adsorbed nematogens. The present investigation closely parallels a previous study ${ }^{12}$ (hereafter referred to as I) of the isotropic-nematic transition of hard ellipsoids. In fact, exactly the same approximate density-functional theory as used in $I$ for $D=3$ will be used here for the $D=2$ case. In this way the influence of the dimensionality $D$ on the results of the density-functional theory can be clearly assessed. In a previous study ${ }^{13}$ it was shown already that the quantitative agreement between the approximate density-functional theory and the computer- 
simulation results for the liquid-solid transition transfers nicely from the hard-sphere to the hard-disk system. In the present case it can only be hoped that the similar agreement found already in I for the isotropic-nematic transition of hard ellipsoids will also be transferred to the hard-ellipse case for which the corresponding computer simulations are, however, still lacking.

In Sec. II we briefly recall the density-functional approach to orientational freezing (as introduced in I) in a $D$-dimensional setting. The results for the isotropic and nematic phases of hard ellipses are given in Sec. III. The relation to the orientational freezing of some constrained bulk systems is briefly considered in Sec. IV, while Sec. V contains our conclusions.

\section{APPROXIMATE DENSITY-FUNCTIONAL THEORY FOR ORIENTATIONAL FREEZING IN $D$ DIMENSIONS}

The density-functional theory (DFT) is a convenient theoretical framework for the study of phase coexistence mainly because it treats the disordered (isotropic) and the ordered (nematic) phases on the same footing. Between the various possible versions ${ }^{11}$ of the DFT we will employ here the one which uses the Helmholtz free energy $F$ as basic thermodynamic potential for reasons already advocated in I. We will follow here the same notation as in I which contains moreover a summary of the general ideas behind the DFT in a setting valid for both positional and orientational freezing. Let $f=F / V$ be the (Helmholtz) free energy per unit volume $V, \beta=1 / k_{B} T$ the inverse temperature (which plays only the role of a scale factor in hard-core systems), and $\rho$ the average (number) density so that $f / \rho$ is the free energy per particle and $\beta f / \rho$ its dimensionless form. Further, $\Delta f=f_{N}-f_{I}$ will denote the difference in free-energy density between the nematic $(N)$ and isotropic $(I)$ phases of the same average density: $\rho_{N}=\rho_{I} \equiv \rho$. The dimensionless free-energy difference, $\beta \Delta f / \rho$, can then be written according to Eq. (2.10) of I:

$$
\begin{aligned}
\frac{\beta}{\rho} \Delta f= & \frac{1}{V} \int d \mathbf{x} \frac{\rho(\mathbf{x})}{\rho} \ln \left(\frac{\rho(\mathbf{x})}{\rho}\right) \\
& -\frac{1}{\rho V} \int d \mathbf{x} \int d \mathbf{x}^{\prime} \int_{0}^{1} d \lambda(1-\lambda) c\left(\mathbf{x}, \mathbf{x}^{\prime} ;[\rho+\lambda \Delta \rho]\right) \\
& \times \Delta \rho(\mathbf{x}) \Delta \rho\left(\mathbf{x}^{\prime}\right)
\end{aligned}
$$

where $\Delta \rho(\mathbf{x})=\rho(\mathbf{x})-\rho$, with $\rho(\mathbf{x})$ the local density at $\mathbf{x}=(\mathbf{r}, \mathbf{u})$ and $c\left(\mathbf{x}, \mathbf{x}^{\prime} ;[\rho]\right)$ the direct correlation function (DCF) of a system of local density $\rho(x)$. Here $r$ labels the position of the center of the molecule and $u$ the orientation $\left(\mathbf{u}^{2}=1\right)$ of its symmetry axis (assumed present) with respect to a laboratory-fixed coordinate system. Restricting ourselves to spatially uniform phases for which $\rho(\mathbf{x})=\rho h(\mathbf{u})$, with $h(\mathbf{u})$ the angular distribution, and using the factorization approximation, ${ }^{12}$

$$
c\left(\mathbf{x}, \mathbf{x}^{\prime}\right)=c_{0}\left(\left|\mathbf{r}-\mathbf{r}^{\prime}\right|\right) \Sigma\left(\mathbf{u} \cdot \mathbf{u}^{\prime}\right),
$$

between the translational and angular variables, Eq. (2.1) is transformed into Eq. (4.7) of I:

$$
\begin{array}{rl}
\frac{\beta}{\rho} \Delta f=\int d \mathbf{u} & h(\mathbf{u}) \ln h(\mathbf{u}) \\
-\frac{\rho}{2} \int d \mathbf{r} c_{0}\left(\frac{|\mathbf{r}|}{\sigma_{0}} ; \bar{\eta}(\eta ; \chi)\right) \\
\quad \times \int d \mathbf{u} \int d \mathbf{u}^{\prime} \Sigma\left(\mathbf{u} \cdot \mathbf{u}^{\prime}\right)[h(\mathbf{u})-1] \\
\times\left[h\left(\mathbf{u}^{\prime}\right)-1\right],
\end{array}
$$

where $\Sigma\left(\mathbf{u} \cdot \mathbf{u}^{\prime}\right)$ is the excluded volume of two molecules of given orientation $\mathbf{u}$ and $\mathbf{u}^{\prime}$, while $c_{0}\left(|\mathbf{r}| / \sigma_{0} ; \eta\right)$ is the DCF of an isotropic system of spherical molecules of diameter $\sigma_{0}$ and of packing fraction $\eta=\rho v_{\text {mol }}$, with $v_{\text {mol }}$ the volume of one molecule, or equivalently of the spherical molecule which serves to model the translational correlations of the original nonspherical molecules (see I for details). Finally, $\bar{\eta}=\bar{\eta}(\eta ; \chi)$ is the packing fraction of the effective isotropic fluid of spherical molecules which is used to model the DCF of the ordered phase of the original nonspherical molecules. As in I, $\bar{\eta}$ will be defined implicitly through the following rescaling of the contact distances:

$$
c_{0}(1 ; \eta)=c_{o}\left[\left(\frac{1-|\chi|}{1+|\chi|}\right)^{1 / 2} ; \bar{\eta}\right],
$$

where $\chi=\left(k^{2}-1\right) /\left(k^{2}+1\right)$ and $k=\sigma_{\|} / \sigma_{\perp}$ is the aspect ratio of the molecule with $\sigma_{\|}\left(\sigma_{\perp}\right)$ being its diameter parallel (perpendicular) to the symmetry axis. All the above approximations are identical to those discussed already in detail in I and have been recalled here mainly to show that they remain valid for a $D$-dimensional system (through appropriate generalization of the vectors $r$ and u). Notice, however, that in the particular case of hard ellipses $(D=2)$ the oblate $(0<k<1)$-prolate $(1<k<\infty)$ symmetry discussed in I degenerates into a trivial statement. This is because ellipses always have two symmetry axes and hence $\mathbf{u}$ can be taken either along the major (prolate) or the minor (oblate) axis. The Berne-Pechukas Gaussian-overlap approximation ${ }^{14}$ to the excluded volume $\Sigma\left(\mathbf{u} \cdot \mathbf{u}^{\prime}\right)$ can also be shown to remain valid for $D$ dimensional ellipsoids:

$$
\Sigma_{\mathrm{BP}}\left(\mathbf{u} \cdot \mathbf{u}^{\prime}\right)=\left(\frac{1-\chi^{2}\left(\mathbf{u} \cdot \mathbf{u}^{\prime}\right)^{2}}{1-\chi^{2}}\right)^{1 / 2}
$$

provided $D \geq 2$. Similarly, for the DCF $c_{0}\left(|\mathbf{r}| / \sigma_{0} ; \eta\right)$ we can use the generalization to $D$-dimensional spheres of the Percus-Yevick hard-sphere DCF elaborated elsewhere. ${ }^{15}$ Finally, for the nematic phase the angular distribution $h(\mathbf{u})$ must be of the form $h(\mathbf{u}) \equiv h(\mathbf{u} \cdot \mathbf{n})$ with $\mathbf{n}$ the director $\left(\mathbf{n}^{2}=1\right)$. For a uniaxial nematic, $\mathbf{n}$ and $-\mathbf{n}$ are equivalent directors so that $h(m)=h(-m)$ with $m=\mathbf{u} \cdot \mathbf{n}$. The parametrization of $h(m)$ used in $I$ is then easily generalized as

$$
h(m)=\exp \left[\sum_{l=0}^{\infty} \gamma_{2 l} C_{2 l}^{(D-2) / 2}(m)\right],
$$

where $C_{l}^{(D-2) / 2}(m)$ are the Gegenbauer polynomials which form a complete basis ${ }^{16}$ for the representation of the group of rotations of $\mathbf{u}$ around the polar axis $\mathbf{n}$ in a 
$D$-dimensional space $(\mathbf{u} \cdot \mathbf{n}=m=\cos \theta)$. The Gegenbauer polynomials $C_{l}^{(D-2) / 2}(x)$ satisfy ${ }^{17}$ the orthogonality condition $(D>1)$

$$
\begin{array}{r}
\int_{-1}^{1} d x\left(1-x^{2}\right)^{(D-3) / 2} C_{l}^{(D-2) / 2}(x) C_{n}^{(D-2) / 2}(x)=0 \\
\text { if } l \neq n
\end{array}
$$

and the symmetry property $C_{l}^{(D-2) / 2}(-x)$ $=(-1)^{l} C_{l}^{(D-2) / 2}(x)$. The one order-parameter $\left(\gamma \equiv \gamma_{2}\right)$ approximation corresponding to Eq. (4.1) of I reads then

$h(m)=\frac{\exp \left[\gamma C_{2}^{(D-2) / 2}(m)\right]}{Z(\gamma)}$,

$Z(\gamma)=\int_{0}^{1} d m \frac{1}{N_{D}}\left(1-m^{2}\right)^{(D-3) / 2} \exp \left[\gamma C_{2}^{(D-2) / 2}(m)\right]$,

where we have put $N_{D}=\Gamma\left(\frac{3}{2}\right) \Gamma[(D-1) / 2] / \Gamma(D / 2)$ with $\Gamma(n+1)=n$ ! the $\Gamma$ function. ${ }^{17}$ The working form of Eq. (2.2) reads now

$$
\begin{aligned}
\frac{\beta}{\rho} \Delta f= & {\left[\gamma \frac{\partial}{\partial \gamma}-1\right) \ln Z(\gamma) } \\
& -\frac{\eta}{2} I(\bar{\eta}(\eta ; \chi))\left[H(\gamma ; \chi)-H_{0}(\chi)\right],
\end{aligned}
$$

with

$$
I(\eta)=\frac{1}{\eta}\left[1-\frac{\partial}{\partial \eta}\left[\eta Z_{0}(\eta)\right]\right),
$$

where $Z_{0}(\eta)=\beta p / \rho$ is the hard $D$-sphere compressibility factor, ${ }^{15}$ and

$$
\begin{aligned}
& H(\gamma ; \chi)=\int d \mathbf{u} \int d \mathbf{u}^{\prime} \Sigma_{\mathrm{BP}}\left(\mathbf{u} \cdot \mathbf{u}^{\prime}\right) h(\mathbf{u}) h\left(\mathbf{u}^{\prime}\right), \\
& H_{0}(\chi) \equiv H(\gamma=0 ; \chi)=\int d \mathbf{u} \int d \mathbf{u}^{\prime} \Sigma_{\mathrm{BP}}\left(\mathbf{u} \cdot \mathbf{u}^{\prime}\right),
\end{aligned}
$$

the average excluded volume of respectively, the nematic and the isotropic phase.

To be more specific we now give some explicit expressions for $D=2$ and $D=3$. For the Gegenbauer polynomials $C_{l}^{(D-2) / 2}(m)$ of $(2.5)$ we have

$$
\begin{aligned}
& C_{l}^{(1 / 2)}(m)=P_{l}(m), \quad D=3 \\
& C_{l}^{(0)}(m)=T_{l}(m), \quad D=2
\end{aligned}
$$

where $P_{l}(m)$ are the Legendre polynomials ${ }^{17}$ and $T_{l}(\cos \theta) \equiv \cos (l \theta)$ the Chebyshev polynomials. ${ }^{17}$ The normalization factor ${ }^{18} Z(\gamma)$ of $(2.7)$ becomes then

$$
\begin{aligned}
Z(\gamma) & =\int_{0}^{1} d m \exp \left[\gamma\left(\frac{3}{2} m^{2}-\frac{1}{2}\right)\right] \\
& =\frac{e^{\gamma}}{\sqrt{(3 / 2) \gamma}} F(\sqrt{(3 / 2) \gamma}), \quad D=3 \\
Z(\gamma) & =\frac{1}{2 \pi} \int_{0}^{2 \pi} d \theta \exp [\gamma \cos (2 \theta)] \\
& =I_{0}(\gamma), \quad D=2
\end{aligned}
$$

where $F(x)$ and $I_{0}(x)$ are, respectively, the Dawson and zeroth-order modified Bessel functions. ${ }^{17}$ The average excluded volume of the isotropic phase, $H_{0}(\chi)$ of (2.11), reads

$$
\begin{aligned}
& H_{0}(\chi)=\frac{1}{2}\left[1+\frac{\arcsin \chi}{\chi\left(1-\chi^{2}\right)^{1 / 2}}\right], \quad D=3 \\
& H_{0}(\chi)=\frac{2}{\pi} \frac{E\left(\chi^{2}\right)}{\left(1-\chi^{2}\right)^{1 / 2}}, \quad D=2
\end{aligned}
$$

where $E\left(\chi^{2}\right)=\int_{0}^{\pi / 2} d \theta\left(1-\chi^{2} \sin ^{2} \theta\right)^{1 / 2}$ is the complete elliptic integral of the second kind. ${ }^{17}$ The average excluded volume of the nematic phase, $H(\gamma ; \chi)$ of $(2.10)$, reads using (2.7)

$$
\begin{array}{r}
H(\gamma ; \chi)=\frac{1}{\left[I_{0}(\gamma)\right]^{2}} \frac{4}{\pi} \int_{0}^{\pi / 2} d \theta\left[\frac{1-\chi^{2} \cos ^{2} \theta}{1-\chi^{2}}\right]^{1 / 2} \\
\times I_{0}(2 \gamma \cos \theta), \quad D=2
\end{array}
$$

whereas the equivalent $D=3$ expression is given by the more complicated Eq. (4.9) of I. Finally the explicit expression used for $c_{o}\left(|\mathbf{r}| / \sigma_{0}, \eta\right)$ in (2.3) is given by Eqs. (3.31) and (3.32) $(D=2)$ and Eq. (3.35) ( $D=3)$ of Ref. 15 together with the corresponding compressibility factors $Z_{0}(\eta)$ of (2.9) for which $Z_{N}(\eta)$ as given by Eq. (2.18) of Ref. 15 was used (with $N=6$ for $D=2$ and $N=2$ for $D=3$ ).

\section{RESULTS OF THE DFT FOR HARD ELLIPSES}

We now consider the results obtained from the general theory proposed in Sec. II in the particular case of hard ellipses $(D=2)$. Unfortunately, we know of very little other data on ellipses to compare with. As far as we know, there are the pioneering Monte Carlo (MC) simulations of Vieillard-Baron ${ }^{19}$ and the theoretical results of Boublik $^{20}$ and of Ward and Lado. ${ }^{21}$

To start, let us consider the isotropic phase where the angular distribution is uniform $[h(\mathbf{u}) \equiv 1]$. In this case the compressibility factor of the hard ellipses, $\beta p / \rho$, can be written using Eq. (3.14) of I:

$$
\frac{\beta}{\rho} p \equiv Z(\eta ; \chi)=1+H_{0}(\chi)\left[Z_{0}(\eta)-1\right],
$$

where $H_{0}(\chi)$ is given now by Eq. (2.17) above. Here $k=\sigma_{\|} / \sigma_{\perp}$ is the aspect ratio of the ellipses and $\chi=\left(k^{2}-1\right) /\left(k^{2}+1\right)$ the related eccentricity variable. From Eq. (3.1) it is seen that the compressibility factor of the hard ellipses of eccentricity $\chi$ and packing fraction $\eta=(\pi / 4) \sigma_{\|} \sigma_{1} \rho, Z(\eta ; \chi)$, is given in terms of the compressibility factor of hard disks, $Z_{0}(\eta)$, of the same area and packing fraction $\left[\eta=(\pi / 4) \sigma_{0}^{2} \rho\right.$ with $\left.\sigma_{0}^{2}=\sigma_{\|} \sigma_{1}\right]$. In other words, Eq. (3.1) states that the dimensionless excess pressures of the corresponding ellipses and disks scale like the respective average excluded volumes:

$$
[Z(\eta ; \chi)-1] /\left[Z_{0}(\eta)-1\right]=H_{0}(\chi) .
$$

As illustrated in Fig. 1, the resulting hard-ellipse equation of state $Z(\eta ; \chi)$ depends slightly on which hard-disk equation of state $Z_{0}(\eta)$ is being used as input in (3.1). In Fig. 2 we compare our results to those obtained by Boub$\mathrm{lik}^{20}$ from the scaled particle theory (SPT) for hard el- 


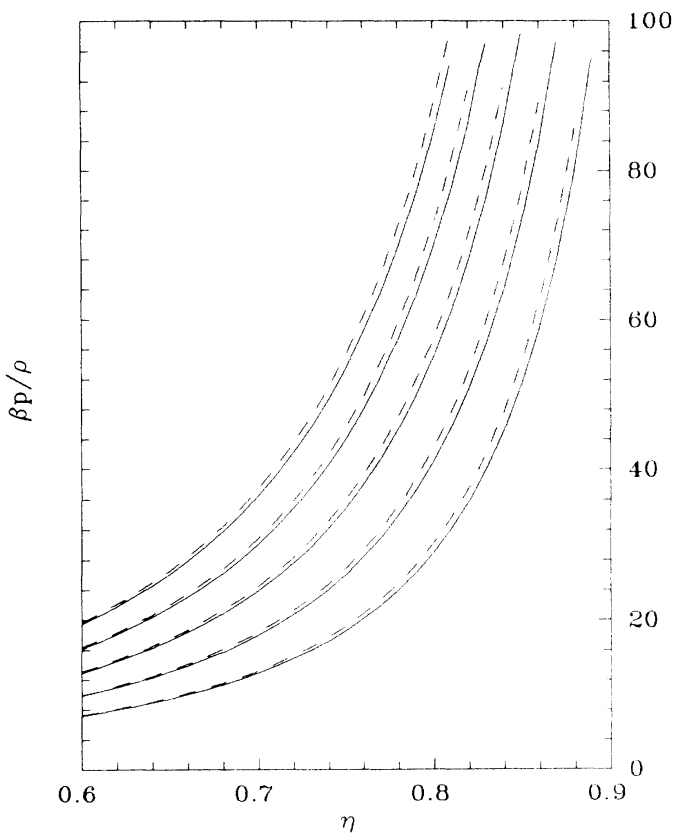

FIG. 1. Compressibility factor $\beta p / \rho$ vs the packing fraction $\eta$ of a system of hard ellipses of aspect ratio $k=2,4,6,8$, and 10 (from bottom to top) as obtained from Eq. (3.1) for two different input hard-disk equations of state: the equation, $Z_{0}=\left(1+\eta^{2} / 8\right) /(1-\eta)^{2}$, proposed by Henderson (Ref. 27) (dashed lines) and the equation proposed by Baus and Colot (Ref. 15) (solid lines). The differences in the resulting hardellipse equations of state (visible only in the high-density region shown here) are comparable to the differences already present (Ref. 15) in the underlying hard-disk equations of state.

lipses. In this case we use the SPT result for hard disks in (3.1), $Z_{0}(\eta)=(1-\eta)^{-2}$, so that both theories will have the same limit as $k$ tends to 1 . Comparing Figs. 1 and 2 we conclude that the theoretical uncertainties in the hard-ellipse equation of state are similar to those of the underlying hard-disk equation of state. While the latter have already been compared to the computer simulations elsewhere, ${ }^{13}$ the corresponding simulations for hard ellipses are unfortunately still lacking.

Next we consider the nematic phase $[h(\mathbf{u}) \neq 1]$ as described by Eqs. (2.7)-(2.11). The effective density, $\bar{\eta}=\bar{\eta}(\eta ; \chi)$, entering Eq. (2.8) is shown in Fig. 3. Its behavior is very similar to that of the $D=3$ case considered in I. The free-energy difference, $\Delta f$ of $(2.8)$, is shown in Fig. 4 as a function of the nematic order parameter $\gamma$ of (2.7). Here the difference with the $D=3$ case of $I$ is quite striking. There is clear evidence that the first-order transition of $I$ has become a continuous transition in $D=2$. Similar evidence for a continuous isotropic-nematic transition in $D=2$ has also been obtained for other systems and by other methods (for a recent discussion see Ref. 9). It is noteworthy, however, that this result is obtained here using exactly the same approximation scheme for $D=2$ as for $D=3$ so that the change from a first-order to a continuous transition can only be ascribed to the change in dimensionality and not to changes in the ap-

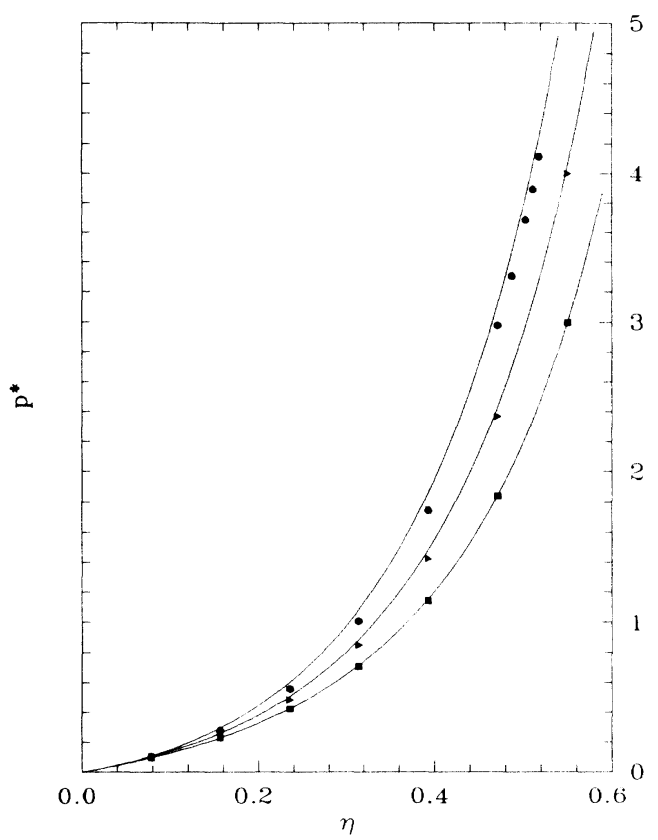

FIG. 2. Reduced pressure $p^{*}=\beta p v_{\text {mol }}$ vs the packing fraction $\eta$ of a system of hard ellipses of aspect ratio $k=2,4$, and 6 (from bottom to top) as obtained from Eq. (3.1) with the SPT hard-disk result $\left[Z_{0}=(1-\eta)^{-2}\right]$ as input (solid lines) and compared to the hard-ellipse SPT results of Boublik (Ref 20) as quoted by Ward and Lado (Ref. 21) (dots). While both theories are identical for $k=1$ the difference between them increases with increasing $k$ value.

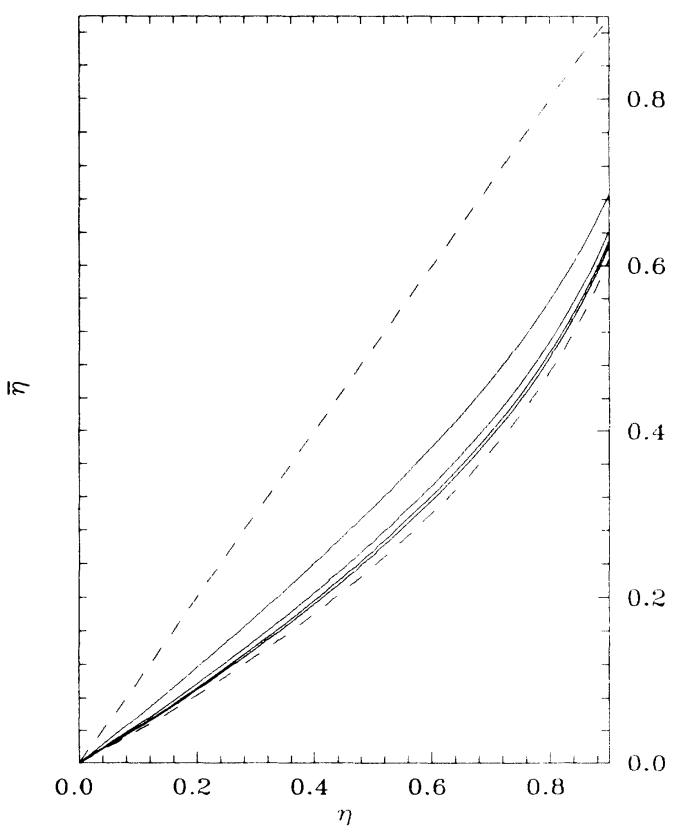

FIG. 3. The relation, Eq. (2.3), between the packing fraction of the nematic $\eta$ and the packing fraction $\bar{\eta}$ of the effective isotropic phase used to approximately describe the correlations in the nematic phase. From top to bottom $k=2,4,6$, and 8 (solid lines). For convenience, the limiting cases $k=1$ and $k=\infty$ have also been included (dashed lines). 


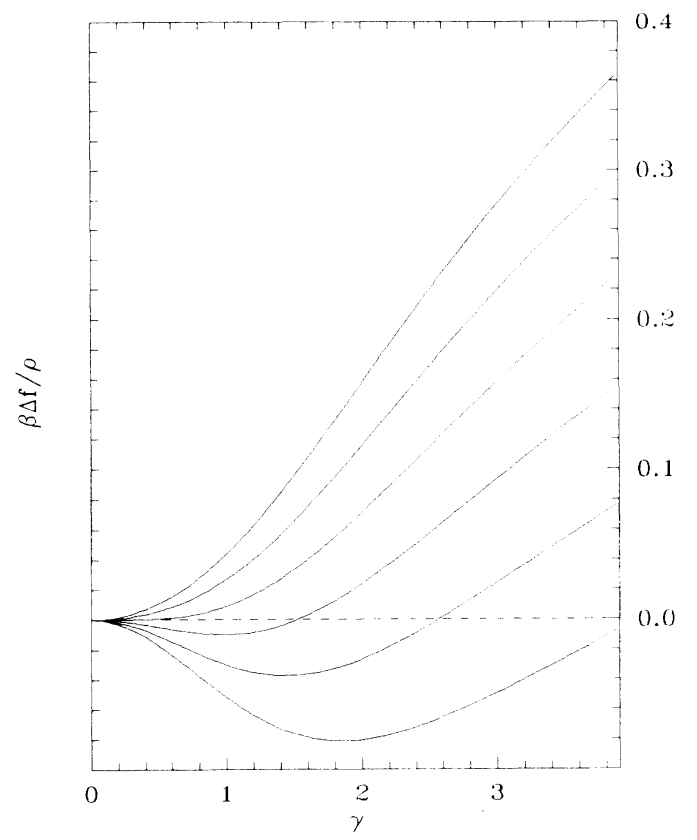

FIG. 4. Reduced free-energy difference $\beta \Delta f / \rho$ between the nematic and isotropic phases of hard ellipses of aspect ratio $k=6$, Eq. (2.8), as a function of the nematic order parameter $\gamma$, Eq. (2.7), for various packing fractions: $\eta=0.29,0.31,0.33$, $0.35,0.37$, and 0.39 (from top to bottom). The critical isochore $\eta^{*} \approx 0.33$ separates the situations where the isotropic phase $\left(\gamma^{*}=0\right)$ is the only free-energy minimum $\left(\eta<\eta^{*}\right)$ from those for which the nematic phase $\left(\gamma^{*} \neq 0\right)$ is the only free-energy minimum $\left(\eta>\eta^{*}\right)$.

proximation scheme. It is clear also that graphically one cannot distinguish between a continuous and a very weak first-order transition. This point is quite pertinent here because the isotropic-nematic transition is already weakly first order for $D=3$. To settle this question we can use a small order-parameter expansion just like in the familiar Landau theory ${ }^{22}$ already discussed in the present context in I. Using the same procedure as in I we first switch from the unbounded order parameter $\gamma$ of (2.7) to the bounded and physically more interesting order parameter $q=q(\gamma)$ :

$$
q=\langle\cos (2 \theta)\rangle \equiv \frac{\partial}{\partial \gamma} \ln Z(\gamma),
$$

where Eq. (2.15) was used. The relation between $\gamma$ $(0<\gamma<\infty)$ and $q(0<q<1)$ is displayed in Fig. 5 . Proceeding as in I we obtain then the following small-q expansion of (2.8):

$$
\frac{\beta}{\rho} \Delta f=\sum_{n=0}^{4} a_{n} q^{n}+O\left(q^{5}\right),
$$

with $a_{0}=a_{1}=a_{3}=0$ and

$$
\begin{aligned}
& a_{2}=1-\eta I(\bar{\eta})\left(A_{2}-A_{0}\right), \\
& a_{4}=\frac{1}{4}-\eta I(\bar{\eta})\left(\frac{1}{3} A_{4}-A_{2}+\frac{1}{4} A_{0}\right),
\end{aligned}
$$

where $\bar{\eta}=\bar{\eta}(\eta ; \chi)$, as above, and $A_{n}=A_{n}(\chi)$ is given by

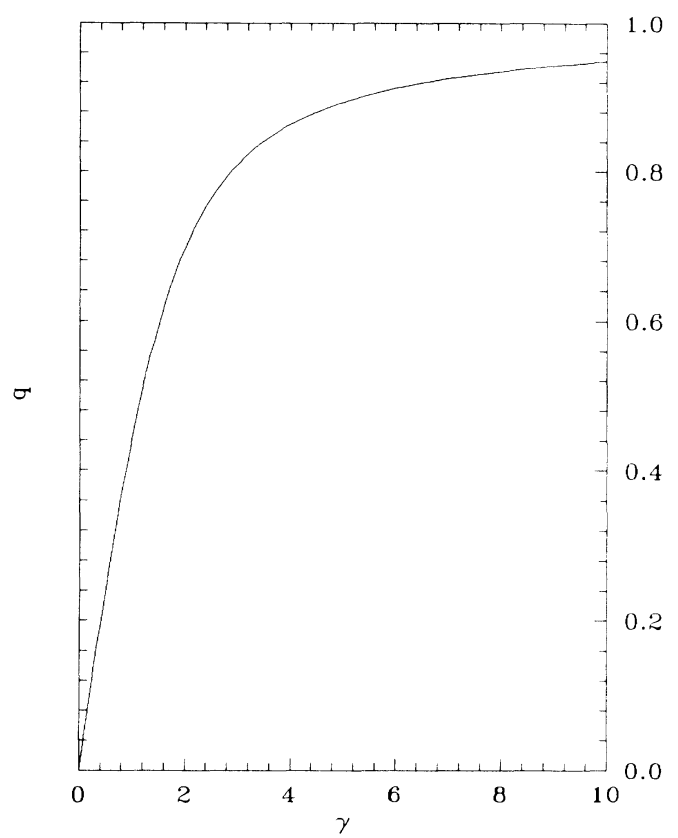

FIG. 5. Relation between the nematic order parameters $\gamma$ of Eq. (2.7) and $q$ of Eq. (3.2).

$A_{2 n}(\chi)=\frac{(2 n) !}{(n !)^{2}} \int_{0}^{2 \pi} d \alpha \frac{1}{2 \pi}\left(\frac{1-\chi^{2} \cos ^{2} \alpha}{1-\chi^{2}}\right)^{1 / 2} \cos ^{2 n} \alpha$

with $n=0,1,2, \ldots$. The fact that in the Landau expansion (3.3) we have $a_{3} \equiv 0$, together with our findings at finite $q$ (see Fig. 4) clearly establishes that in the present case $(D=2)$ the isotropic-nematic transition predicted by this theory is continuous. For $D=2$ one can also show that the free energy (2.8) is, in fact, an even function of $q$ (or $\gamma$ ), i.e., $a_{2 n+1} \equiv 0$ for all $n=0,1,2, \ldots$ in (3.3), whereas for general $D$ one can show that the coefficient $a_{3}$ of (3.3) behaves like

$$
a_{3} \sim\{\Gamma[(D-2) / 2]\}^{-4},
$$

and hence $a_{3}$ approaches zero continuously when $D$ reaches the value 2 from above. The value $D=2$ appears thus as a marginal value for which the generally firstorder transition $(D>2)$ becomes continuous $(D=2)$.

This point being settled, we return now to the general properties of the isotropic-nematic transition in $D=2$. If we denote by $q^{*}$ the value of the order parameter $q$ for which, at a given value of $\chi$ and $\eta$, the free energy attains its minimum, then $q^{*}=q^{*}(\eta ; \chi)$ tends continuously to zero when $\eta$ is decreased from the high-density region down to a critical value $\eta^{*}$ at which $q^{*}$ vanishes $\left[q^{*}\left(\eta^{*} ; \chi\right)=0\right.$ for $\left.\eta^{*}=\eta^{*}(\chi)\right]$. For $\eta \leq \eta^{*}$, the freeenergy minimum remains at $q^{*}=0$, corresponding to the isotropic phase $\left(q^{*}=0\right)$ being the only stable phase, whereas for $\eta>\eta^{*}$ the nematic phase $\left(q^{*} \neq 0\right)$ is the only stable phase. There are no metastable phases here; the extrapolation of (3.1) into the region $\eta>\eta^{*}$ corresponds 
thus to an unstable isotropic phase. It is easily shown that, in the vicinity of $\eta^{*}$ and for all $\chi$ values, $q^{*}$ behaves like $q^{*} \sim\left(\eta-\eta^{*}\right)^{1 / 2}$ for $\eta \geq \eta^{*}$. Such a classical criticalpoint behavior was to be expected here since we made no attempt to incorporate order-parameter fluctuations ${ }^{22}$ into the theory. The location of the critical point $\eta^{*}(\chi)$ is very sensitive to the value of $\chi$. For very elongated ellipses one can show from (3.3) that $\eta^{*}$ tends to zero as $\eta^{*} \sim 3 \pi / 4 k$, for $k \rightarrow \infty$. In the opposite limit of weak eccentricity $\eta^{*}$ is instead pushed into the high-density region according to $1-\eta^{*} \sim(k-1)^{2 / 3}$, for $k \rightarrow 1$. This behavior together with the intermediate values of $\eta^{*}$ is further illustrated in Fig. 6. It should also be remembered here that in the high-density region the isotropic-nematic transition may be preemptied by other transitions not taken into account here. For instance, for $1<k<2.5, \eta^{*}$ exceeds the value $(\eta=0.690)$ at which hard disks $(k=1)$ will freeze. ${ }^{13}$

Having located the stable phases we can now construct the full equation of state of the nematic phase. The simplest procedure here is to use Eq. (2.2) of Ref. 23, which in the present approximation reads

$$
\begin{aligned}
\frac{\beta}{\rho} p_{N}(\eta ; \chi)= & \frac{\beta}{\rho} p_{I}(\eta ; \chi) \\
& -\frac{\eta}{2} I(\bar{\eta}(\eta ; \chi))\left[H\left(\gamma^{*}(\eta ; \chi) ; \chi\right)-H_{0}(\chi)\right],
\end{aligned}
$$

where $\gamma^{*}$ denotes the value of $\gamma$ corresponding to $q^{*}$ [i.e., $q^{*}=q\left(\gamma^{*}\right)$ using (3.2), although in practice $q^{*}$ is deduced from $\gamma^{*}$ which is obtained directly by minimization of the free energy] and $p_{N}$ is the pressure of the

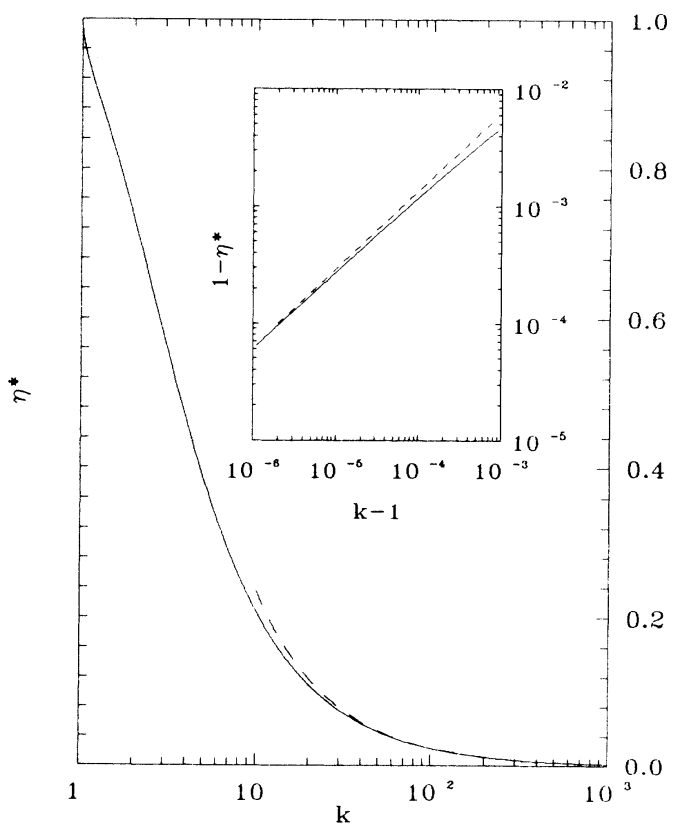

FIG. 6. Critical packing fraction $\eta^{*}$ vs the aspect ratio $k$ as obtained from Eq. (3.4) (solid lines) and the asymptotic behavior discussed in the main text (dashed lines). nematic phase while $p_{I}$ is the pressure of the isotropic phase as given by Eq. (3.1). Notice that in (3.7), Eq. (3.1) has to be evaluated in the region $\eta>\eta^{*}$ where the isotropic phase is thermodynamically unstable. From Figs. 1 and 2 it is seen that this offers no particular difficulty. In Fig. 7 we compare the results obtained from Eqs. (3.7) and (3.1) to the MC simulations of Vieillard-Baron ${ }^{19}$ and to the numerical solution of the Percus-Yevick equation as obtained by Ward and Lado. ${ }^{21}$ While the overall behavior of the various results is quite similar our comparison calls nevertheless for some remarks. In the computer simulations of Vieillard-Baron a very weak first-order isotropic-nematic transition was observed at a density considerably higher than our $\eta^{*}$ which corresponds to a continuous transition. It is well known that VieillardBaron's pioneering simulations exhibit a large amount of scatter and his results have already been criticized by Frenkel and Eppenga. ${ }^{9}$ It is clearly advisable to redo ${ }^{9}$ these simulations before drawing a definite conclusion. Some reservations should also be made with respect to our comparison with the results of Ward and Lado. ${ }^{21}$ Indeed, while their equation-of-state data exhibit no tran-

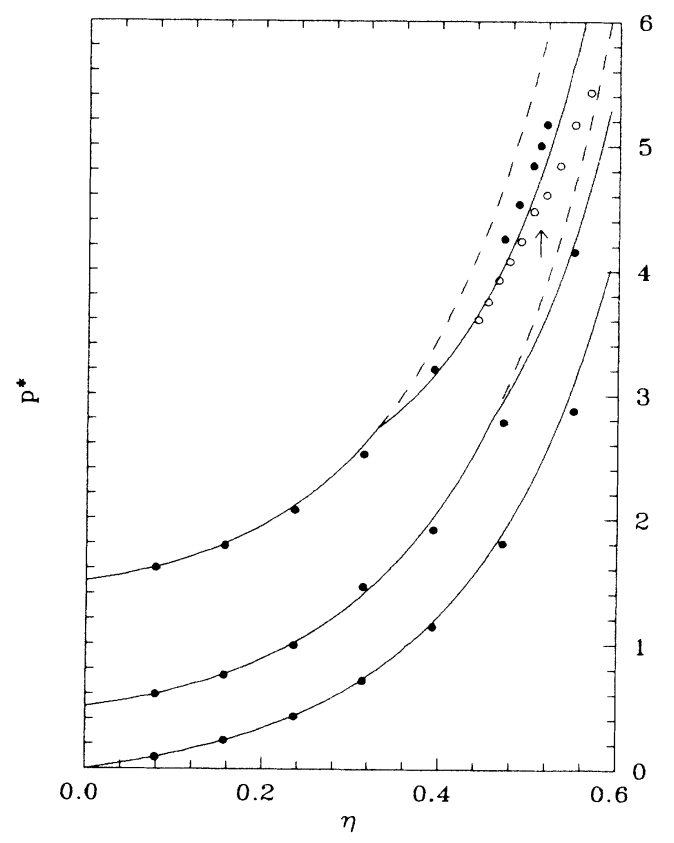

FIG. 7. Reduced pressure $p^{*}=\beta p v_{\mathrm{mol}}$ vs the packing fraction $\eta$ of hard ellipses of aspect ratio $k=2,4$, and 6 (from bottom to top) as obtained from the present theory [Eqs. (3.1) and (3.7); solid lines for the stable isotropic and nematic phases, dashed lines for the unstable isotropic phase] and compared to the MC-simulation results of Vieillard-Baron (Ref. 19) ( $k=6$, open circles) and to the numerical solution of the Percus-Yevick equations by Ward and Lado (Ref. 21) (closed circles). The arrow indicates the density for which Vieillard-Baron $(k=6)$ found a weakly first-order transition, while here we find a continuous transition at $\eta^{*}=0.33$ for $k=6$ and $\eta^{*}=0.46$ for $k=4$, whereas Ward and Lado found no transition (see, however, main text for important reservations). [To separate the curves, the $p^{*}$ scales have been shifted as $p^{*}(k=2) p^{*}+0.5(k=4)$, and $p^{*}+1.5(k=6)$.] 
sition but are otherwise comparable to ours it should be remembered here that these authors have solved numerically the Percus-Yevick equations for hard ellipses under the assumption that the system is uniform and isotropic (hence $\gamma=0$ ) but allowing for long-range angular pair correlations characteristic of the nematic phase. It is not obvious how this is to be related to our definition of a nematic as a uniform system with an anisotropic angular distribution $(\gamma \neq 0)$.

In the limit of very elongated or equivalently of very thin ellipses $(k \rightarrow \infty$ or $k \rightarrow 0$ ) our system should behave similarly to the hard-needle system considered by Frenkel and Eppenga. ${ }^{9}$ As we have seen above, $\eta^{*}$ vanishes in this limit and we may expect the virial expansion of the free energy (2.8) to be a reasonable approximation. As discussed already in I such a virial expansion will produce an Onsager-type theory. ${ }^{13,24}$ For $D=2$ the corresponding Onsager theory turns out to be particularly simple. Indeed, taking the limit $k \rightarrow \infty, \eta \rightarrow 0$ and introducing the Onsager variable $c=(2 / \pi) \eta k$ (which is kept finite) Eqs. (2.8) and (3.7) reduce simply to

$$
\begin{aligned}
&\left.\frac{\beta}{\rho} \Delta f\right|_{\text {Ons }}= {\left[\gamma \frac{I_{0}^{\prime}(\gamma)}{I_{0}(\gamma)}-\ln I_{0}(\gamma)\right) } \\
&\left.-c \mid 1-\frac{\int_{0}^{1} d t I_{0}(2 \gamma t)}{\left[I_{0}(\gamma)\right]^{2}}\right), \\
&\left.\left.\frac{\beta}{\rho} p\right|_{\text {Ons }}=1+c-c \mid 1-\frac{\int_{0}^{1} d t I_{0}\left(2 \gamma^{*}(c) t\right)}{\left[I_{0}\left(\gamma^{*}(c)\right)\right]^{2}}\right),
\end{aligned}
$$

where $I_{0}(\gamma)$ is the zeroth-order modified Bessel function, $I_{0}^{\prime}(\gamma) \equiv I_{1}(\gamma)$ its derivative, ${ }^{17}$ and $\gamma^{*}(c)$ the value of $\gamma$ obtained by minimization of (3.8). Before attempting a direct comparison with the hard-needle system ${ }^{9}$ one has to take into account that hard ellipses have always a finite radius of curvature whereas hard needles (considered as hard rectangles of zero width) have not. This geometric difference gives both systems slightly different packing properties. A meaningful comparison between the ellipses and the needles can be made, in the Onsager limit, if we compare systems with the same second virial coefficients, $B_{2}=\sigma_{\|}^{2} / 2=L^{2} / \pi$, where $\sigma_{\|}$is the major diameter of the ellipses and $L$ the length of the needles. Indeed, as seen from (3.8) and (3.9), the only thing which matters in the Onsager limit is the rescaled density variable $c$ which can also be written $c=B_{2} \rho$. In Fig. 8 we compare the results of (3.8) and (3.9) to the computer simulations of Frenkel and Eppenga ${ }^{9}$ for hard needles of length $L=\sigma_{\|} \sqrt{\pi / 2}$. The agreement between theory and simulations is quite satisfactory. Both approaches yield a continuous isotropic-nematic transition with a good agreement for the critical point $c^{*}=\frac{3}{2}$.

\section{RESULTS FOR A CONSTRAINED SYSTEM OF ELLIPSOIDS}

It has become customary in the literature to consider systems where some of the motions are rigorously constrained to a subspace. For example, one has studied ${ }^{25}$

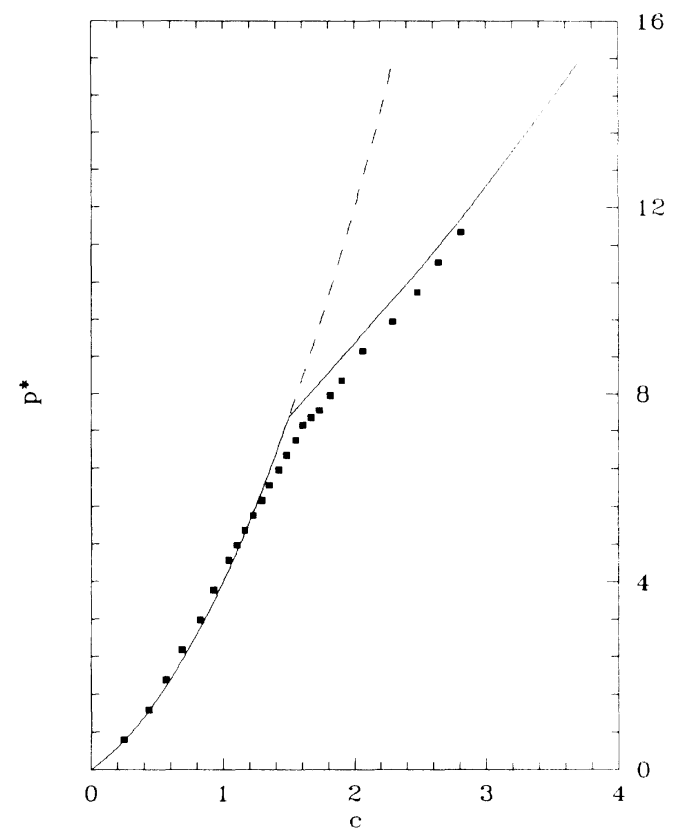

FIG. 8. Reduced pressure $p^{*}=\beta p \sigma_{\|}^{2}$ vs the Onsager variable $c=(2 / \pi) \eta k$ as obtained from Eqs. (3.8) and (3.9) (solid lines: stable isotropic and nematic phases; dashed line: unstable isotropic phase) and compared to the constant-pressure MCsimulation results (squares) of Frenkel and Eppenga (Ref. 9) for a system of 200 hard needles of length $L=\sigma_{\|} \sqrt{\pi / 2}$, so that both systems will have the same second virial coefficient. The low-density isotropic branch is well approximated by $p^{*}=2 c(1+c)$, and the high-density nematic branch by $p^{*}=4 c$. The theoretical critical point is $c^{*}=\frac{3}{2}$.

the smectic ordering of spherocylinders which remain strictly parallel one to each other, or else the nematic ordering of hard rods which are grafted on a plane. ${ }^{26} \mathrm{Be}-$ sides being easier to simulate, such systems are also expected to mimic the behavior of the original system under the influence of very strong (in principle, infinite) external constraints (e.g., electric or magnetic fields). Within the present approximate DFT it is fairly easy to study such constrained systems. This is due to the fact that within the present approximation the angular and positional variables are completely factorized. To study constrained systems it is sufficient then to distinguish the dimensionality of the spatial variable $\mathbf{r}$, say $D$, from the dimensionality of the angular variable $\mathbf{u}$, say $d$, and reduce the value of $D$ or $d$ to the dimensionality of the subspace allowed by the given constraint. For instance, ellipsoids grafted on a plane would correspond to $D=2$ and $d=3$, or else parallel ellipsoids will correspond to $D=3$ and $d=1$. This then immediately raises the question of how the phase transitions known to exist in the original system will be affected by the introduction of such a constraint. Above we have seen that the orientational freezing of hard ellipsoids $(D=d=3$ ) differs qualitatively from the orientational freezing of hard ellipses $(D=d=2)$ in that the former is first order while the latter is continuous. From the present discussion we can 


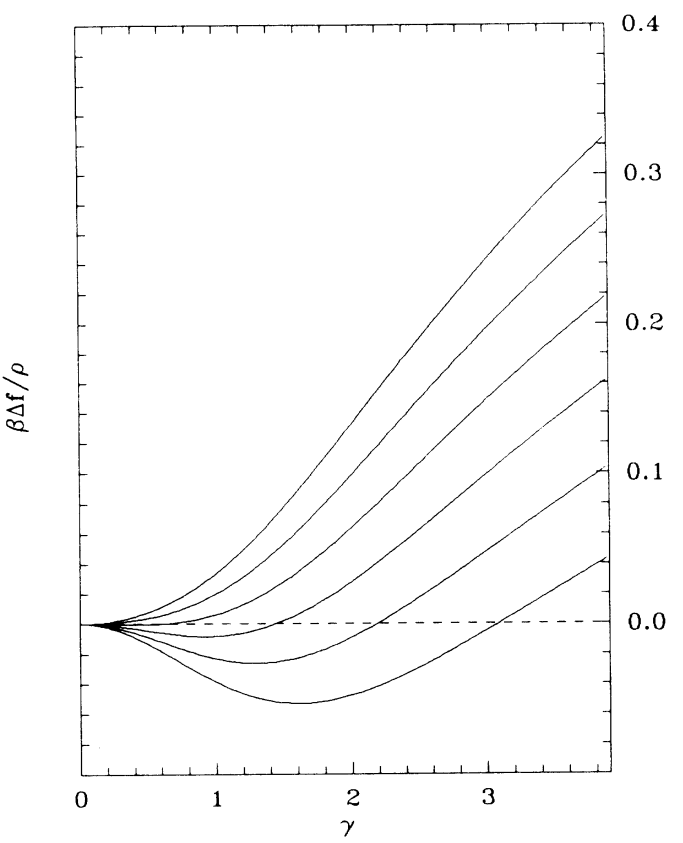

FIG. 9. Same as in Fig. 4 but for a $(D=3)$ system of hard ellipsoids $(k=6)$ whose angular motions are constrained to remain perpendicular to a given direction. The packing fractions are here $\eta=0.17,0.18,0.19,0.20,0.21$, and 0.22 (from top to bottom) and the critical value $\eta^{*} \approx 0.19$.

conjecture that the nature of the orientational freezing transition (continuous or first order) will be determined solely by the value of $d(d=2$ or $d>2)$ whatever the value of $D$. It should be clear, however, that the location of the transition $\left(\eta^{*}\right)$ may still depend on the value of $D$.

To illustrate this we now consider a system of hard ellipsoids $(D=3)$ whose symmetry axes $(\mathbf{u})$ are constrained to remain orthogonal to a given direction, or equivalently remain parallel to a given plane, reducing thereby the value of $d$ from 3 to 2 . The general theory of Sec. II can be applied immediately to this constrained system by introducing only minor modifications. For the angular distribution $h(\mathbf{u} \cdot \mathbf{n})$ we can still use the parametrization given by Eq. (2.7) with the understanding that the director $\mathbf{n}$ has now to be taken perpendicular to the direction of the constraint. The fact that we are considering ellipsoids $(D=3)$ instead of ellipses $(D=2)$ enters now through the fact that the DCF $c\left(|\mathbf{r}| / \sigma_{0} ; \eta\right)$ used in Eqs. (2.2) and (2.3) has to describe hard spheres $(D=3)$ instead of hard disks $(D=2)$. This can be achieved, for example, by using the well-known ${ }^{15}$ Percus-Yevick hardsphere DCF for it. As seen from Fig. 9 and expected from the above conjecture, the orientational freezing of the constrained hard ellipsoids proceeds now through a continuous transition and not through a first-order transition as found in I for the unconstrained system. The critical point $\eta^{*}$ is, however, shifted to considerably lower values of $\eta$ (see Fig. 10). From the same conjecture we also conclude that the orientational freezing of ellipsoids grafted on a plane $(D=2, d=3$ ) will be first order. This then immediately raises the question of what the

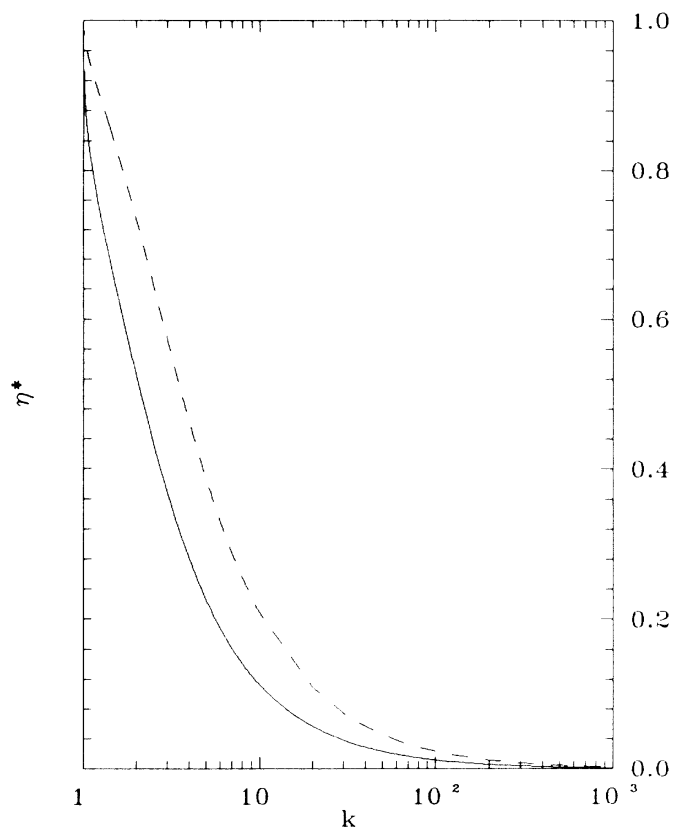

FIG. 10. Critical packing fraction $\eta^{*}$, for the continuous isotropic-nematic transition as a function of the aspect ratio $k$ for a $D=2$ system of hard ellipses (dashed line) and a $D=3$ system of constrained hard ellipsoids with two-dimensional angular motions (solid line).

transition should look like for a real nematogen adsorbed on a real substrate. Indeed, if the molecules stick strongly to the substrate they should behave like the hard ellipses, whereas if they have a tendency to "stand up" they should behave more like the grafted ellipsoids.

\section{CONCLUSIONS}

We have proposed an approximate density-functional theory of orientational freezing in $D$ dimensions. The theoretical results obtained from this theory are, as shown in I, in good agreement with the computer simulations of the isotropic-nematic transition of hard ellipsoids $(D=3)$. Here we have presented the corresponding results for hard ellipses $(D=2)$ for which no extensive simulation results exist as yet. The most striking difference between the two systems is the change in nature of the isotropic-nematic transition which proceeds through a continuous transition for the ellipses while it proceeds through a first-order transition for the ellipsoids. We have also shown that, within this theory, the width of the generally first-order orientational freezing transition shrinks continuously to zero when the dimensionality of the angular space is reduced continuously towards the marginal value of 2 (from above), whatever the value of the dimensionality of the positional space. The latter point has important implications for the study, within this theory, of the orientational freezing transition in systems subject to a constraint which restrains the angular motions of the molecules. 


\section{ACKNOWLEDGMENTS}

One of us (M.B.) acknowledges the support of the Association EURATOM-Etat Belge and also of the Fonds
National de la Recherche Scientifique. This work has, moreover, been partially sponsored by the Comisión Asesora de Investigación Científica y Técnica (Spain) Project No. PB85-0024.
${ }^{1}$ P. G. de Gennes, The Physics of Liquid Crystals (Clarendon, Oxford, 1974); G. Vertogen and W. H. de Jeu, Thermotropic Liquid Crystals: Fundamentals (Springer-Verlag, Berlin, 1988).

${ }^{2}$ For a recent review see, e.g., D. Frenkel, J. Phys. Chem. 92, 3280 (1988).

${ }^{3}$ See, for example, B. H. Mulder and D. Frenkel, Mol. Phys. 55, 1193 (1985); U. P. Singh and Y. Singh, Phys. Rev. A 33, 2725 (1986); M. Baus, J. L. Colot, X. G. Wu, and H. Xu, Phys. Rev. Lett. 59, 2184 (1987).

${ }^{4}$ W. Maier and A. Saupe, Z. Naturforsch., Teil A 13, 564 (1958); W. L. McMillan, Phys. Rev. A 4, 1238 (1971).

${ }^{5}$ J. P. Hansen and I. R. McDonald, Theory of Simple Liquids (Academic, London, 1976); D. A. McQuarrie, Statistical Mechanics (Harper and Row, New York, 1976).

${ }^{6}$ See, for example, K. J. Strandburg, Rev. Mod. Phys. 60, 161 (1988).

${ }^{7}$ Another example of reduced dimensionality is given by the freely suspended liquid-crystal films studied by C. Y. Young, R. Pindak, N. A. Clark, and R. B. Meyer, Phys. Rev. Lett. 40, 773 (1978).

${ }^{8}$ For a recent discussion closely related to the present topic see the introduction of D. H. Van Winkle and N. A. Clark, Phys. Rev. A 38, 1573 (1988). See also J. P. Straley, Phys. Rev. A 4, 675 (1971).

${ }^{9}$ D. Frenkel and R. Eppenga, Phys. Rev. A 31, 1776 (1985).

${ }^{10}$ See D. H. Van Winkle and N. A. Clark, Phys. Rev. A 38, 1573 (1988).
${ }^{11}$ For recent reviews see, e.g., A. D. J. Haymet, Annu. Rev. Phys. Chem. 38, 89 (1987); M. Baus, J. Stat. Phys. 48, 1129 (1987).

${ }^{12}$ J. L. Colot, X. G. Wu, H. Xu, and M. Baus, Phys. Rev. A 38, 2022 (1988) (referred to as I).

${ }^{13}$ J. L. Colot and M. Baus, Phys. Lett. A 119, 135 (1986).

${ }^{14}$ B. J. Berne and P. Pechukas, J. Chem. Phys. 56, 4213 (1972).

${ }^{15}$ M. Baus and J. L. Colot, Phys. Rev. A 36, 3912 (1987).

${ }^{16}$ N. Ja. Vilenkin, Fonctions Spéciales et Théorie de la Représentation des Groupes (Dunod, Paris, 1969).

${ }^{17}$ Handbook of Mathematical Functions, edited by $\mathbf{M}$. Abramowitz and I. A. Stegun (Dover, New York, 1965).

${ }^{18}$ The normalization factor, $Z(\gamma)$ of $(2.7)$, should not be confused with the compressibility factor, $Z_{0}(\eta)$ of (2.9).

${ }^{19}$ J. Vieillard-Baron, J. Chem. Phys. 56, 4729 (1972).

${ }^{20}$ T. Boublik, Mol. Phys. 29, 421 (1975).

${ }^{21}$ D. A. Ward and F. Lado, Mol. Phys. 63, 623 (1988).

${ }^{22}$ L. Landau and E. Lifchitz, Physique Statistique (Mir, Moscow, 1984).

${ }^{23}$ J. L. Colot and M. Baus, Mol. Phys. 56, 807 (1985).

${ }^{24}$ See also R. F. Kayser and H. J. Raveché, Phys. Rev. A 17, 2067 (1978).

${ }^{25}$ A. Stroobants, H. N. W. Lekkerkerker, and D. Frenkel, Phys. Rev. A 36, 2929 (1987).

${ }^{26}$ Z. Y. Chen, J. Talbot, W. M. Gelbart, and A. Ben-Shaul, Phys. Rev. Lett. 61, 1376 (1988).

${ }^{27}$ D. Henderson, Mol. Phys. 30, 971 (1975). 